\title{
Considerations from the Epicenter of the Coronavirus Emergency
}

\author{
${ }^{1}$ Fondazione Ospedale Maggiore Policlinico IRCCS Cà Granda, Milan, \\ Italy \\ 2JROP Institute of Echocardiography, US and Vascular Doppler, \\ Delhi, India \\ ${ }^{3}$ Internal Medicine Unit, Ospedale di Crema, CR, Italy \\ J Card Crit Care:2020;4:64-65
}

Fabiola B. Sozzi ${ }^{1}$ Rakesh Gupta ${ }^{2}$ Ciro Canetta ${ }^{3}$ Francesco Blasi ${ }^{1}$

\begin{abstract}
Address for correspondence Fabiola Sozzi, MD, PhD, Ospedale Maggiore Policlinico IRCCS, Milan, Italy

(e-mail: fabiola_sozzi@yahoo.it).
\end{abstract}

\begin{abstract}
Keywords

- biological catastrophe

- coronavirus disease

- COVID 19
\end{abstract}

Since February 21, 2020, Lombardy region in northern Italy has experienced an outbreak of coronavirus disease 2019 (Covid-19), caused by the severe acute respiratory syndrome coronavirus 2 (SARS-CoV-2). This region, mainly a flat land with the highest density of population and industry of the country, has become the single deadliest hotspot for the coronavirus on the planet so far. More than 10,000 people have died here, with the national total across the country poised to top 18,000 . The World Health Organization (WHO) first declared the Covid-19 respiratory illness unknown before the outbreak reported in Wuhan, China, last December. On January 30, 2020, the WHO denounced the Covid-19 outbreak a global health emergency. Some scientists declared the virus began circulating in Italy at least a month prior to the first case of infection recorded in February.

\section{Introduction}

Since February 21, 2020, Lombardy region in northern Italy has experienced an outbreak of coronavirus disease 2019 (Covid-19), caused by the severe acute respiratory syndrome coronavirus 2 (SARS-CoV-2). This region, mainly a flat land with the highest density of population and industry of the country, has become the single deadliest hotspot for the coronavirus on the planet so far. More than 10,000 people have died here, with the national total across the country poised to top 18,000 . The World Health Organization (WHO) first declared the Covid-19 respiratory illness unknown before the outbreak reported in Wuhan, China, last December. On January 30, 2020, the WHO denounced the Covid-19 outbreak a global health emergency. Some scientists declared the virus began circulating in Italy at least a month prior to the first case of infection recorded in February.

I am working as a clinical cardiologist in a tertiary hospital of Milan. We realized the epidemic state when the hotbed was already and irredeemably diffused in the area. The medical services of Lombardy have been suddenly overwhelmed by acute presentations of patients with Covid- 19 . Most of the hospitals of the area have been rapidly converted into Covid clinics; also, the cardiology unit where I work had the same destination. Our staff have been running "intensive training" sessions to prepare for how to manage the particular needs of Covid-19 patients in a middle-high intensity care unit. In a few days, hospitals of the north were overrun and there were no free intensive care beds in the Lombardy region. This will impact our ability to deliver acute and elective care to patients with chronic diseases such as cardiovascular diseases. A marked reduction in acute admissions of patients with acute coronary syndromes, heart failure, and so forth have been recorded. Oral, nasal, or nasopharyngeal swabs have been used among symptomatic patients arriving in the hospital and healthcare workers with respiratory symptoms and/or fever. Considering that some asymptomatic patients may be a source of infection and transmission, all patients started to be managed as suspected cases of Covid-19. At all times, patient transfer to different areas of the hospital was minimized to reduce the risk for infection transmission. Clinical priorities and procedures have changed during this pandemic. As cardiologists, we learned that cardiac imaging in patients with suspected or confirmed Covid-19 should be
DOI https://doi.org/

10.1055/s-0040-1710402

ISSN 2457-0206.
License terms

() (1) $\ominus \circledast$ 
performed if appropriate, and only if it is likely to substantially change patient management or be lifesaving. Elective, nonurgent and routine follow-up examinations should be postponed or even cancelled. Echocardiography should, therefore, generally be reserved for patients with symptomatic heart disease.

The Italian government dealt with the pandemic by issuing a series of decrees that gradually increased restrictions within lockdown areas ("red zones"), which were then expanded until they were ultimately applied to the entire country. In the population and also in many policy circles, an initial resistance was encountered toward the declared state of emergency, although several scientists had been warning of the potential of a catastrophe for weeks. Each region of the country has released different policies to define who is expected to undergo the Covid-19 testing in order to detect the viral genome. Such a nonuniform approach even within the country consequently generated different rates, possibly uncertainness with regard to its spread in the population, and higher risk of dismal outcomes. The hospitals were rapidly populated by infected patients, facilitating transmission to uninfected patients. The initial epidemiologic research suggests that asymptomatic spreading is happening. Up to $25 \%$ of people infected may never have symptoms. And in others, their symptoms may not show up until 48 hours after being infected, according to new evidence. Yet, researchers have said people in both groups may be "silent spreaders" of the virus. A study on transmission in Singapore and China found that infection is transmitted between 2.55 and 2.89 days, respectively, before symptoms starts. ${ }^{1}$ Therefore, we deduce that hospitals might be the main Covid-19 carriers and health workers can be asymptomatic carriers or sick without surveillance. A recent study from the University of Nebraska Medical Center found widespread evidence of viral RNA in isolation rooms of patients being treated for Covid-19. ${ }^{2}$ A consistent number of healthcare workers have died so far, increasing the stress especially of those on the front line. Certainly, major deadly diseases have an unpredictable power.
Our experience suggests a general lack expertise on epidemic conditions to rapidly and effectively absorb and act upon epidemiological knowledge. The fact that the quarantine rules have not been followed since the beginning has helped to spread the disease. Considering that some asymptomatic patients may be a source of infection and transmission, all patients should be managed as suspected cases of Covid-19, recommending an "infected until proven otherwise" approach. Health workers need surveillance, especially for avoiding the carrier condition. What this teaches is the need to guide us to adopt special measures in order to reduce epidemiologically negative behaviors in the outbreak.

\section{Conclusion}

The massive overload of the healthcare structures represents a medical and economic shock. It is unquestionably Italy's biggest crisis since World War II. The high-fatality rate of this severe and highly contagious pneumonia exterminated entire families in some areas of Lombardy, the most affected region of the country. We learned how important is the mass testing, tracing, and isolating the infected once. Clinical priorities and procedures have changed during this pandemic. The isolation with loneliness extends beyond Covid patients and reminds the pages of Manzoni, an Italian writer, who touchingly described the plague in Milan during the 1600. Indeed, to face and manage an outbreak is extremely complex and confirms the vulnerability of human beings.

\section{References}

1 TindaleL,CoombeM,StockdaleJ,etal.Transmissionintervalestimates suggest pre-symptomatic spread of COVID-19. MedRxiv (e-pub ahead of print ). doi 10.1101/2020.03.03.20029983

2 Santarpia JL, Rivera DN, Herrera V, et al. Transmission potential of SARS-CoV-2 in viral shedding observed at the University of Nebraska Medical Center. MedRxiv (e-pub ahead of print) doi 10.1101/2020.03.23.20039446 